\title{
Editorial
}

\section{Special issue on networked system control and connected vehicles: opportunities and challenges}

CASE (Connected, Automated, Sharing, and Electrifying) is a global trend in the automotive industry due to the big potential in improving energy efficiency and reducing the air pollution from automobile exhaust. Indeed, the connectivity, connecting the vehicles with the internet, is firstly implemented in the automotive industry in the sense of large scale connection of vehicles. The connected environment has been two decades in the automotive industry which enables us to provide a much comfortable and smart telemetric service. However, the attention has not been focused on the control technology with the connectivity for efficiency and emission improvement. From the view of system control, the connected vehicles are large-scaled, multi-agent or high dimension systems that coupled and interacted but centralized control is not reasonable. How to formulate the optimization or control problem for the connected vehicles and how to solve the problem with system control theory are significant challenging issues.

This special issue collected seven papers that addressed these control problems from the view of networked system and optimal control theory. The collection can be divided into three groups.

The first group includes three papers that focused on vehicle control with the use of $\mathrm{V} 2 \mathrm{~V}$ and $\mathrm{V} 2 \mathrm{I}$ information. The article by Qiuyi Guo et al., demonstrated the possibility of improving the fuel economy of fuel cell trucks using the traffic light signal. It is shown that with the V2I information, the model predictive control technology can save more than $\mathbf{7 . 4 3 \%}$ hydrogen consumption in a case study driving cycle. The model predictive control technology is also applied to car-following control on an urban road network by using V2V and V2I information. The paper by A. S. M. Bakibillah et al., investigated this issue and it is shown that the control with V2Vand V2I can improve traffic flow and fuel economy. The paper by Bo Zhang et al., proposed a two-stage optimization approach for speed planning and energy management of hybrid electric vehicles, where the control policy of MPC is fully applied in the two stages of design and a typical scenario of merging is targeted.

The second group collected two papers that focused on automated driving. For automated vehicles, control of vehicle dynamics is the main subject, but it is an important elemental subject for driving vehicles under connected environment. Controlling an individual vehicle in the scene of parking is addressed in the paper by Dequan Zen et al. which also demonstrated real test results. The issue of driving-by-wire full is investigated in the paper by Ping Wang et al., where again MPC is exploited for developing the real-time control law.

Finally, two articles are collected that discussed active fault tolerant control for connected mobile robots by $\mathrm{M}$. Hussein et al., and powertrain control for electric vehicles with robust control theory by J. Buerger ad J. Anderson, respectively.

(c) 2019 South China University of Technology, Academy of Mathematics and Systems Science, CAS and Springer-Verlag GmbH Germany, part of Springer Nature 
We hope this special issue will provide a new inspiring for those working in the front of both optimization of networked system and control of connected vehicle systems. We wish to thank Yiguang Hong, Editor of Journal of Control Theory and Technology, for his timely organization of this special issue.

\section{Guest editors:}

Hong Chen

Tongji University, Shanghai, China

Tielong Shen

Sophia University, Tokyo, Japan 